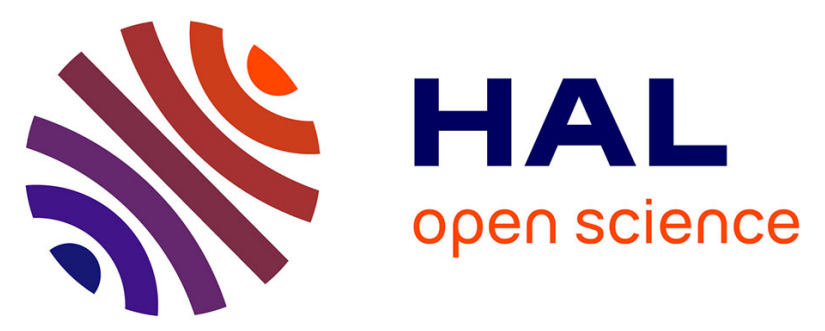

\title{
Using BV-norm to Classify the Vasculitis in Multiple Sclerosis Fundus Angiography for Ophthalmologists
}

Giang Bang Nguyen, Daniel Delahaye, Stéphane Puechmorel, P. Marechal, P. Olle

\section{- To cite this version:}

Giang Bang Nguyen, Daniel Delahaye, Stéphane Puechmorel, P. Marechal, P. Olle. Using BV-norm to Classify the Vasculitis in Multiple Sclerosis Fundus Angiography for Ophthalmologists. Journal of Medical and Bioengineering, 2013, 2013 International Conference on Functional Biomedical Imaging (ICFBI), 2 (1), pp.11-15. 10.12720/jomb.2.1.11-15 . hal-00918054

\section{HAL Id: hal-00918054 \\ https://hal-enac.archives-ouvertes.fr/hal-00918054}

Submitted on 9 Jan 2017

HAL is a multi-disciplinary open access archive for the deposit and dissemination of scientific research documents, whether they are published or not. The documents may come from teaching and research institutions in France or abroad, or from public or private research centers.
L'archive ouverte pluridisciplinaire HAL, est destinée au dépôt et à la diffusion de documents scientifiques de niveau recherche, publiés ou non, émanant des établissements d'enseignement et de recherche français ou étrangers, des laboratoires publics ou privés. 


\title{
Using BV-norm to Classify the Vasculitis in Multiple Sclerosis Fundus Angiography for Ophthalmologists
}

\author{
B. G. Nguyen, \\ Institut de Math_ematiques de Toulouse (IMT). \\ Email: bgnguyen@math.univ-toulouse.fr \\ D. Delahaye and S. Puechmorel \\ Ecole national de l'Aviation Civile (ENAC). \\ Email: daniel@recherche.enac.fr \\ P. Marechal \\ ISAE-SUPAERO \\ P. Olle \\ Chu de Toulouse
}

\begin{abstract}
Retinal diseases are causing alteration of the visual perception leading sometimes to blindness. For this reason early detection and diagnosis of retinal pathologies is very important. Using digital image processing techniques, retinal images may be analyzed quickly and computerassisted diagnosis systems may be developed in order to help the ophthalmologists to make a diagnose. This paper describes the method using BV norm for classification of vasculitis fundus angiography. We propose a method to help ophthalmologists for the diagnosis of such autoimmune disease. Our study aimed to deter-mine potential angiography details in intermediate uveitis helping to diagnose multiple sclerosis (MS).
\end{abstract}

Index Terms - vasculitis fundus angiography, multiple sclerosis

\section{INTRODUCTION}

Intermediate and/or posterior uveitis could preceded one set of MS in 30-46\% of the patients [1]. The reported frequency of uveitis varies from $0.4 \%$ to $26.9 \%$ in multiple sclerosis patient [2]. But, the prevalence of multiple sclerosis in patients with uveitis is $1-2 \%$. Frequently it is difficult for an ophthalmologist to diagnose MS when the patient start with vasculitis. It takes many years (8-9 years) until neurological symptoms help to diagnose MS. It seems in series that the prognosis of such uveitis is not so well known (visual acuity, disability) and some controversy [3]. Our aim was first to analyse the angiography of patients who started the disease by intermediate uveitis and diagnosed as MS. With these results, we analysed angiography of patients with auto immune intermediate uveitis presumed as MS.

Manuscript received Nov 2, 2012; revised Jan 3, 2013.

\section{A. Periphlebitis Vasculitis and Multiple Sclerosis}

Intermediate and posterior uveitis are the most frequent form of uveitis [4]. The potential risk of developing neurological disease is $60 \%$ in 5 years when there is a association of periphlebitis and optic neuropathy as $16 \%$ when there is only optic neuropathy [5]. This association is reported in $28 \%$ of the cases which are near the frequency of uveitis in Multiple sclerosis. Periphlebitis are histologically constitued by in ammatory cells in the edge of the retinal to venous. In experimental auto immune encephalopathy models, such periphlebitis were described in the area adjacent to the de-myelinated lesions [6]. The authors hypothetised that there is an autoimmune reaction to a commune antigen [7].

\section{B. Vasculitis: 3 Types}

Active form: vascular sheathing not well limited with irregular caliber of the vessels. They are situated at a distance from the optic nerve. They could persist from few months to two years.

A cicatricial form or venous sclerosis: the lesions are fixed, linear, distributed by segments and situated in the medium and peripheral retina. Some have a variable constriction of the vascular diameter.

A form with a reduction of the venous caliber.

\section{Intermediate Uveitis and Pars-planitis.}

Gross pathologic examination of the peripheral "snow bank" in parsplanitis shows exudate deposited on the peripheral retina and parsplana. The histology reveals a collapsed vitreous, blood vessels, broglia cells including fibrous astrocytes, and scattered in ammatory cells. Peripheral veins show lymphocytic cuffing and infiltration. The vascular component of the snowbank is continuous with the retina [8]. Pars planitis is a primary 
peripheral perivascularitis and not a choroiditis. Once begun, the process of vascular occlusion may lead to vitritis and snowbank formation and in ammation of the adjacent tissue area by breakdown of the blood-ocular barrier [9], [10].

Following is some illustrating pictures. In the Fig. 1, the sheathing is demonstrated by red arrow whereas the Fig. 2 illustrating a normal retinal image.

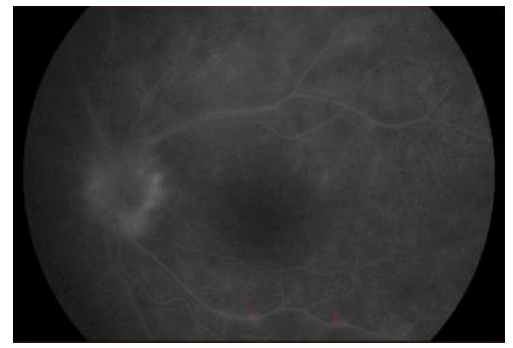

Figure 1. Picture illustrating sheathing of the vessel edges

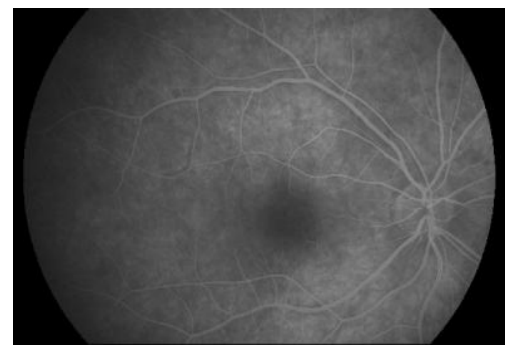

Figure 2. Picture illustrating normal retinal image

\section{Methods}

Patient population and data.

Patients are registered at the Toulouse clinic ophthalmologic and neurological department since 2000. All started the disease by Uveitis. It is a retrospective data collection. Group 1: 30 patients with MS and 5 patients with a presumed SEP (uncompleted criterias). The SUN classification was used for intermediate uveitis [11] and for neurological MS the Mc-Donald criteria 2001 revised 2005 was used [12]. Groups Controls was made with normal Angiography and from patients with others autoimune deseases (sarcodosis, behcet, MICI ...).

\section{Detection And Measurement of Blood Vessels IN RETINAL IMAGES.}

Blood vessels on the retina can be directly captured non-invasively through pupil, in vivo. Vessel appearance is an important indicator for diagnosing different diseases.

Abnormalities of retinal vasculatures can indicate health problem. Vasculature response to different physiological pathologies has been widely studied using variety of methods. Detection and measurement is usually the first step for detection of pathology on digital retinal image. The retinal vessels are the only part of the central circulation that can be viewed directly and studied in detail [13]. Detection and measurements of vessel calibre have been widely studied using a variety of methods. Measurements have been made both directly using ophthalmoscopy and indirectly using fundus photographs.
Segmentation of vessels and measurement of the vascular diameter are two critical and challenging technical tasks in any system attempting an automated diagnosis of vascular conditions. A number of methods for segmenting vascular network have been re-ported in the literature [16], [17], with some success at least for larger vessels, although many papers gloss over the difficulty of reliably segmenting vessels in images of diseased retina, where there are significant distractors; robust segmentation therefore remains an important research issue. There are several authors who have presented algorithms for measuring vascular diameters. Brinchmann-Hansen et al [14], [15] measured widths of retinal vessels using micro densitometry. They presented an algorithm called Full Width Half Maximum (FWHM) to measure vascular diameters. This approach calculates a "half height point" on the left and right sides of the initial estimated mid-point of the profile. On each side, the minimum and maximum intensity levels are calculated, and the "half height point" is located where the profile crosses the mid-point with intensity between minimum and maximum. The FWHM estimate of the profile width is then the distance between these half height points. This approach is also called Half Height Full Width (HHFW).

Gregson et al [18] introduced an alternative approach, including a rectangular profile that is fitted to the profile data. The height of this rectangle is fixed to the difference between the minimum and maximum intensity values in the profile. The width of the rectangular profile is adjusted until the area under the rectangular pro le is equal to the area under the profile data. Chapman et al [19], compares three methods of automated vascular measurements with manual recorded vessel diameters. A Gauss function using non-linear regression, a Sobel edge detection algorithm and a sliding linear regression filter (SLRF) are all compared.

Chapman states that using the Sobel edge detection algorithm to locate the blood vessel edges is unreliable due to a tendency to wrongly locate the central light re ex as the vessel edge. Chapman found the SLRF method to be the most accurate and robust of these three methods; however, it is not adopted for low-resolution images, as a minimum number of 10 pixels are required to estimate the slope by linear regression.

In our research, we choose the method of the authors Bankhead P et al [20] to get the center line of the vessels and the diameters. The authors present a novel algorithm for the efficient detection and measurement of retinal vessels. Their algorithm is general enough so it can be applied both low and high resolution fundus photographs and fluorescein angiograms upon the adjustment of only a few intuitive parameters. The algorithm described is fully automated analysis the retinal vessel diameters. It allows the fastest diameters computation all along the length of each vessel rather than at specific points of interest.

\section{Classification using SupPort Vector MachineS}

The support vector machines were first suggested by Vapnik in the 1960s for classification and have recently become an area of intense research. SVM is a training 
algorithm for learning classification and regression rules from data, for example the SVM can be used to learn polynomial, radial basis function (RBF) and multi-layer perceptron (MLP). Nowadays, SVMs become a popular classification technique. A classification task usually involves separating data into training and testing sets. Each instance in the training set contains one "target value" (i.e the class labels) and several "attributes" (i.e. the features or observed). The goal of SVM is to produce a model (based on the training data) which predicts the target values of the test data given only the test data attributes. In this context, we do not go into the details of analysis, we only outline of SVMs.

\section{A. Support Vector Machines}

Given a training set of instance-label pairs $\left(x_{i} ; y_{i}\right), i=1$, $\cdots, 1$ where $x_{i} \in \mathbf{R}^{\mathbf{n}}$ and $\mathrm{y} \in\{-1 ; 1\}$ (Here is two-class case, the k-class case can easily be extended), the support vector machines require the solution of the following optimization problem:

$$
\begin{gathered}
\min _{\omega, b, \xi} \frac{1}{2} \mathrm{w}^{\mathrm{T}} \mathrm{w}+C \sum_{i=1}^{l} \xi_{i} \\
\text { subject to } y_{i}\left(\mathrm{w}^{\mathrm{T}} \phi\left(x_{i}\right)+b\right) \geq 1-\xi_{i} \\
\xi_{i} \geq 0,
\end{gathered}
$$

Here, training vectors $\mathbf{x}_{\mathrm{i}}$ are mapped into a higher (may be infinite) dimensional space by the function. SVM nds a linear separating hyperplane with the maximal margin in this higher dimensional space. $C>0$ is the penalty parameter of the error term. Furthermore $\mathrm{K}\left(\mathbf{x}_{\mathrm{i}} \mathbf{x}_{\mathrm{j}}\right)\left(\mathbf{x}_{\mathrm{i}}\right)^{\mathrm{T}}\left(\mathbf{x}_{\mathrm{j}}\right)$ is called the kernel function. The functions below are the commonly basic used kernels:

- linear: $K\left(\mathrm{x}_{i} ; \mathrm{x}_{j}\right)=\mathrm{x}^{\mathrm{T}}{ }_{i} \mathrm{x}_{j}$

- polynomial: $K\left(\mathrm{x}_{i} ; \mathrm{x}_{j}\right)=\left(\gamma \mathrm{x}_{i}^{\mathrm{T}} \mathrm{x}_{j}+r\right)^{d} \gamma>0$.

- $\quad$ radial basis function $(\mathrm{RBF})$ :

$$
K\left(\mathrm{x}_{i} ; \mathrm{x}_{j}\right)=\exp \left(-\gamma\left\|\mathrm{x}_{i}-\mathrm{x}_{j}\right\|^{2}\right), \gamma>0
$$

- $\quad$ sigmoid: $K\left(\mathrm{x}_{i} ; \mathrm{x}_{j}\right)=\tanh \left(\gamma \mathrm{x}_{i}^{\mathrm{T}} \mathrm{x}_{j}+r\right), \gamma>0$.

Here $\gamma, r$, and $d$ are kernel parameters.

3.2 In the guide to Support vector Classification the authors in [22] point out scaling before applying SVM is very important. The main advantage ò scaling is to avoid attributes with large numeric ranges to dominate over those with smaller numeric ranges. Another advantage is to avoid numerical difficulties during the computation.

\section{Calculation of BV noRm Along Centrelines AND ITS PRESENTATION ON REAL DATA}

The total variation has been introduced in Computer Vision first by Rudin, Osher and Fatemi [21], as a regularizing criterion for solving inverse problems. It has been proven to be quite efficient for regularizing images without smoothing the boundaries of the objects. Normally, one can apply it for solving differential equations as well for image processing.
In this paper the total variation method was applied for measuring the change of image intensity. This method can be used because image is blurred alongside the blood vessel if disease occurs in this blood vessel (See Fig. 1). We calculate BV norm at each point on centrelines in circle area or in rectangular area.

\section{A. Notations and Preliminary Remarks}

Let $X$ denote the Euclidean space $\mathbb{R}^{\mathrm{N} \times \mathrm{N}}$. To de ne the discrete total variation, we introduce a discrete (linear) gradient operator. If $\mathrm{u} \in \mathrm{X}$, the gradient $\nabla u$ is a vector in $\mathrm{Y}=\mathrm{X} \times \mathrm{X}$ given by

$$
(\nabla u)_{i, j}=\left((\nabla u)_{i, j}^{1},(\nabla u)_{i, j}^{2}\right)
$$

with

for $i, j=1, \ldots, N$.

$$
\begin{aligned}
& (\nabla u)_{i, j}^{1}= \begin{cases}u_{i+1, j}-u_{i, j} & \text { if } i<N \\
0 & \text { if } i=N\end{cases} \\
& (\nabla u)_{i, j}^{2}= \begin{cases}u_{i, j+1}-u_{i, j} & \text { if } j<N \\
0 & \text { if } j=N\end{cases}
\end{aligned}
$$

Then, the total of variation of $u$ is defined by

$$
J(u)=\sum_{1 \leq i, j \leq N}\left\|(\nabla u)_{i, j}\right\|
$$

with $\|y\|=\sqrt{y_{1}^{2}+y_{2}^{2}}$ for every $y \in \mathbb{R}^{2}$. In our strategy we calculate the total variation on two different domains (circle and rectangular), which is described in Fig. 3 and in Fig. 4 is the following.

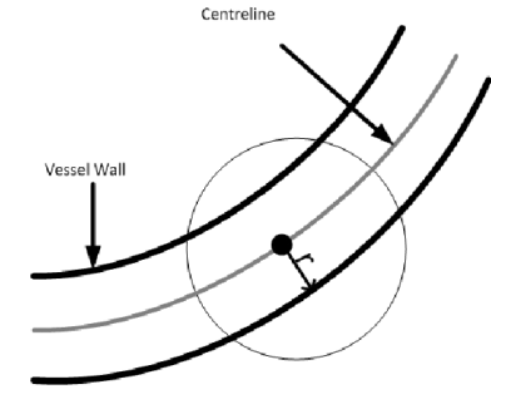

Figure 3. BV norm computation on circle domain

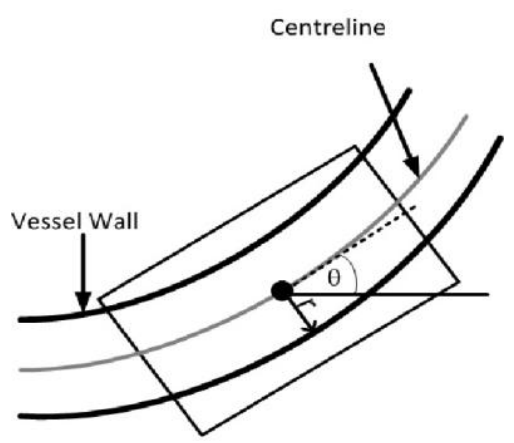

Figure 4. BV norm computation on rectangular domain

In the case circle domain, at each point $\mathrm{P}$ on the centrelines, total variation is given by 


$$
J(u)=\sum_{i, j \in I}\left\|(\nabla u)_{i, j}\right\|
$$

with $I$ is circle centre $P$ and radius $R,(R>r$ which is determined by experiment), $\mathrm{r}$ is the radius of blood vessel at $P$. by

In the case rectangular domain, total variation is given

$$
J(u)=\sum_{i, j \in D} \|(\nabla u)_{i, j}
$$

where $D$ is the rectangular with centre $P$, and width $w>2 r$ which will be defined by experiment. $D$ is rotated a angle (see Fig. 4).

\section{B. The Results are Demonstrated by Color Map}

After computing BV norms at each point of the vessel centrelines, we normalize them via dividing by the area on which the BV norm is calculated. To demonstrate with color map, we choose the range of colours including [Cyan; Green; Blue; Yellow; Pink; Orange; Magenta; Red; Maroon]. This range is ordered from light to dark. All of BV norm is regrouped corresponding to the number of colours. The experimental results which experiment on some diseased images shown that, the in aimed vessels branches manifest with the colour is darker (See the below figures, Fig. 5 and Fig. 6, diseased vessels is localized by red circle).

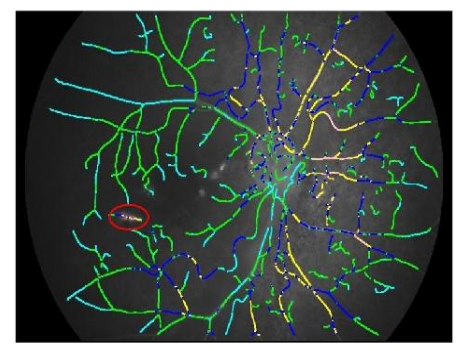

Figure 5. Region manifest disease in the red circle

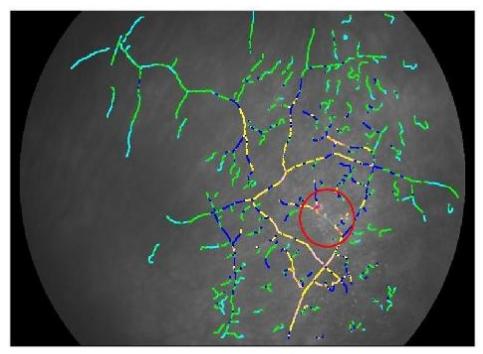

Figure 6. Region manifest disease in the red circle

\section{Histogram}

Then, we create histograms from the experiment on both of the diseased images and non-diseased images. The range $\left[\mathrm{min}_{\mathrm{BV} \text { norm }} ; \max _{\mathrm{BV} \text { norm }}\right]$, is divided equally into $\mathrm{nb}$ intervals. And then we count points on centrelines corresponding to BV norms in each interval. The number of points in each interval is normalized via dividing by the total points of centrelines. Those histogram will be used for classification between disease cases and non disease cases. The next pictures (See Fig. 7-10) are histograms of two diseased images and two non-diseased images.

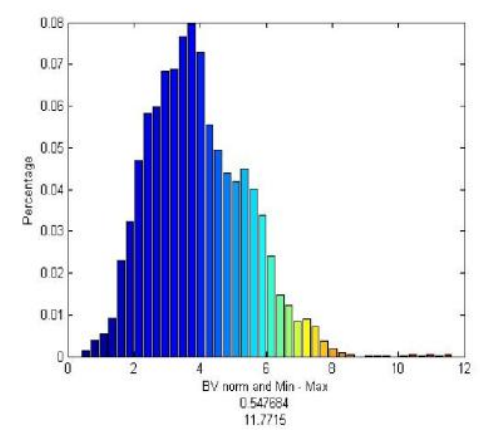

Figure 7. Histogram of a normal case

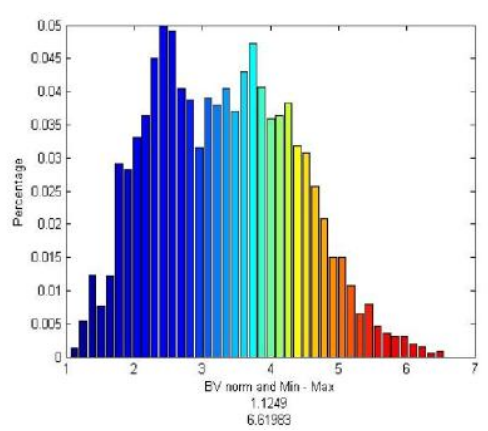

Figure 8. Histogram of a diseased case

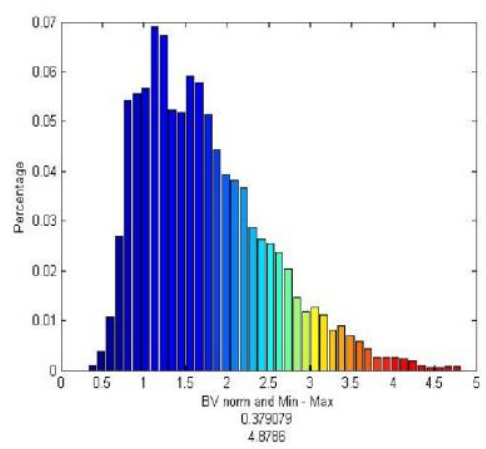

Figure 9. Histogram of a diseased

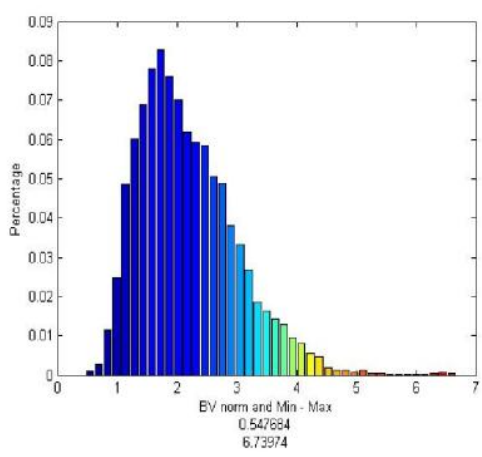

Figure 10. Histogram of a normal case

V. EXPERIMENTAL RESULTS 


\section{A. Data}

Because there are not public data of vasculitis images, we thanks an ophthalmologist to support both diseased images and non-diseased images (41 images). The diseased images are marked by ophthalmological expert. After that we have divided randomly it into two sets, one set for training and one set for testing. The training set includes 21 images and the test set includes 20 images.

\section{B. Result}

At first, all pictures are processed by using the Bankhead $\mathrm{P}$ algorithm to get characteristics of vessels (centrelines, radius, angles). All results are stored in les (*.dat). Then, we compute the BV norm along the centrelines with various sizes of regions. After that, we divide $\mathrm{BV}$ norm range $\left[\mathrm{min}_{\mathrm{BV}}\right.$ norm; $\max _{\mathrm{BV}}$ norm] into $\mathrm{nb}$ intervals, where $\mathrm{nb}$ is considered as a parameter. Points on centrelines corresponding to $\mathrm{BV}$ norm in each interval are counted and then are normalized by dividing the total points of centrelines. The out-put consists of two text les (test le and training le) and they contain nb features for each image.

To classify, we use the libsvm library from ChihChung Chang and Chih-Jen Lin, version 3.12 [22]. By changing various parameters, the algorithm achieved accuracy of $80 \%(16=20)$

\section{CONCLUSION}

This paper has presented a method classification kind of retinal disease. We have tested 41 images and the result we get is $80 \%$ of good classification. In the experiment, we calculated BV norm on different domains (Circle and Rectangular) but we have not yet concluded which case shows the best result. In the future, we hope to receive more pictures for testing and we expect to improve accuracy in image classification.

\section{REFERENCES}

[1] Julie Le Scan et al, "Uveitis associated with multiple sclerosis," Multiple sclerosis 2007; 00:1-3

[2] N. Marchomichelakis "Multiple sclerosis in Foster S and itale A," (eds). Diagnosis and treatment of uveitis. philadelphia: WB. Sanders company, 2002.

[3] Schmidt S et al . Journal of the Neurological Sciences, 2001; 15 : 49-53; Multiple sclerosis 2007; 00:1-3.

[4] J. F. Jordan et al., "Intermediate uveitis in childhood preceding the diagnosis of multiple sclerosis," Am ophthalmol, vol. 135, pp. 8856, 2003.
[5] M. K. Birch et al., "Retinal venous sheathing and blood-retinal barrier in multiple sclerosis," Arch Ophtalmol, vol. 114, pp. 34-39. 1996

[6] D. M. Bachman et al., "Granulomatous uveitis in neuro-logical disease," Br J Ophtalmol, vol. 69, pp. 192-196, 1985.

[7] M. Patt et al., "Vascularites rtiniennes prolifrantes et sclrose en plaque," J Fr Ophtalmmol, vol. 26, no. 4, pp. 381-385, 2003.

[8] J. E. Pederson et al. "Pathology of parsplanitis," Am J Ophtalmol, pp. 762-774, 1986

[9] A. K. Vine et al., "Severe periphlebitis, peripheral retina ischemia, and preretinal neovascularization in patients with multiple sclerosis," Am . J Ophtalmol, vol. 113, pp. 28-32, 1992.

[10] Nussenblatt RB et al., "Fundamentals and linical practice," 2 ed . St Louis: Mosby, 199; pp. 354-363.

[11] "Standardization of Uveitis Nomenclature for reporting clinical data," Am J Ophtalmol, vol. 140, pp. 509-516, 2005.

[12] D. Miller et al., "Clinically isolated syndromes suggestive of multiple sclerosis, part I : Natural history, pathogen-esis, diagnosis, and prognosis," Lancet; vol. 4, pp. 281-288, 2005.

[13] S. M. B. Rammm V. Patel, O. Brinchmann-Hansen, O. Engvold, and E. M. Kohner, "Accurate vessel width measurement from fundus photographs: A new concept," British Journal of Ophthalmology, vol. 78, pp. 24-29, 1994.

[14] O. Brinchmann-Hansen and O. Engvold, "Micropho-tometry of the blood column and light streak on retinal vessels in fundus photographs," Acta Ophthalmologica Supplement, vol. 179, pp. 919, 1986.

[15] O. Brinchmann-Hansen and H. Heier, "Theoretical rela-tionships between light streak characteristics and opti-cal properties of retinal vessels," Acta Ophthalmological Supplement, vol. 179, pp. 33-37, 1986

[16] A. Hoover, V. Kouznetsova, and M. Goldbaum, "Locating the blood vessel in retinal images by piecewise threshold probing of a matched lter response," IEEE Transactions on Medical Imaging, vol. 19, pp. 203-310, 2000.

[17] S. Chaudhuri, S. Chatterjee, N. Katz, M. Nelson, and M. Goldbaum, "Detection of blood vessels in retinal images using two-dimensional matched lters," IEEE Transactions on Medical Imaging, vol. 8, pp. 263-369, 1989.

[18] P. H. Gregson, Z. Shen, R. C. Scott, and V. Kozousek, "Automated grading of venous beading," Computers and Biomedical Research, vol. 28, pp. 291-304, 2000.

[19] N. Chapman, N. Witt, X. Goa, A. Bharath, A. V. Stan-ton, S. A. Thom, and A. D. Hughes, "Computer algo-rithms for the automated measurements of retinal ar-teriolar diameters," British Journal of Ophthalmology, vol. 85, pp. 75-79, 2001.

[20] P. Bankhead, C. N. Schol eld, J. G. McGeown, and T. M. Curtis "Fast retinal vessel detection and measurement using wavelets and edge location re nement," Flos One vol. 7, no. 3, e32435. 2012. 10.1371/journal.pone.0032435,

[21] L. I. Rudin, S. Osher, and E. Fatemi, "Nonlinear total variation based noise removal algorithms," Physica D, vol. 60, pp. 259-268, 1992.

[22] C. C. Chung and L. C. Jen, "A library for support vector machines," ACM Trans-Actions on Intelligent Systems and Technology, vol. 2, pp. 1-27, 2001. 